\title{
Violencia Familiar En Una Empresa Mexicana Del Sector Maquilador
}

\author{
Patricia A. Araiza Zapata, M.S.I. \\ Mario Carrera Ramos, Dr. \\ Alma Rosa Araiza Zapata , M.A. \\ Universidad Autonóma de Chihuahua.
}

doi: 10.19044/esj.2016.v12n23p11 URL:http://dx.doi.org/10.19044/esj.2016.v12n23p11

\begin{abstract}
In the actuality the violence against women can produce a big amount of negative effects in which they bring the decrease or as well the destruction of labor productivity, as it may generate invisible costs for the organizations. The objective of the research is in order to determinate the viability of implementing a internal supportive program, aimed to woman that are victims of domestic violence in an industrial company of Chihuahua City, and being in the possibility of minimizing the labor consequences of the women affected by domestic violence. The applied methodology was made with a design research no experimental, transversal and with mixed nature, as it counted with the appliance of a measure instrument that consists in a survey where the resulting information was that inside of the organization theres a lot of women that has been part or are part of domestic violence. It was also found that $73 \%$ of female staff operating level primarily suffer from domestic violence, these women match on the need to implement support programs, which would be of great benefit to them in their personal and working life. The impact created at work can be seen reflected in: delays, truancy, permits, rescinding his contract , shift change, labor accidents and disclaimers. The domestic violence that could be presented in different ways either : sexual, physical, psychological or financial.
\end{abstract}

Keywords: Domestic violence, productivity

\section{Resumen}

En la actualidad la violencia en contra las mujeres produce en ellas una serie de efectos negativos los cuales a su vez trae consigo la disminución o bien destrucción de la productividad laboral, ya que genera costos invisibles para las organizaciones. El objetivo del presente es con la finalidad de determinar la viabilidad de implementar un programa de apoyo 
interno, dirigido a las mujeres víctimas de violencia familiar en una empresa del sector industrial de la $\mathrm{Cd}$. de Chihuahua, y estar en la posibilidad de minimizar las consecuencias laborales de la mujer violentada. La metodología aplicada fue con un diseño de investigación no experimental, transversal y de naturaleza mixta. Se utilizó un instrumento de medición consistente en un cuestionario aplicado únicamente a mujeres que laboran dentro de dicha organización tanto de nivel operativo como administrativo, en donde se confirmó la hipótesis planteada en lo referente a las consecuencias de la mujer violentada, así como el hecho que se ve impactada por dicho hecho la productividad de la empresa analizada. Así mismo se encontró que un $73 \%$ del personal femenino principalmente de nivel operativo sufre de violencia familiar, dichas mujeres coinciden en la necesidad de implementar programas de apoyo, que les resultaría de gran beneficio tanto en su vida personal como laboral. En la afectación de su trabajo se puede ver reflejado en: retardos, faltas injustificadas, permisos, recisión de contrato, cambio de tuno, accidentes laborales y renuncias. La violencia familiar que se pude presentar de diferentes formas ya sea: sexual, física, psicológica o financiera

Palabras clave: Violencia familiar, productividad

\section{Introducción}

La violencia es un problema social que afecta diversas esferas de nuestra vida. De manera cada vez más evidente, todos estamos expuestos a la violencia y todos podemos generarla en distintas formas. (Secretaria de Educación Pública, 2000)

La violencia es un acto social y en la mayoría de los casos un comportamiento aprendido en un contexto permeado por inequidades sociales basadas en el género, la edad, la raza, etc., con imágenes de violencia y fuerza física como la manera prevaleciente de resolver conflictos. (Cervantes-Sánchez et. al., 2016)

En este sentido el tema de la violencia ejercida en este caso en contra de la mujer, se puede decir que se trata de una problemática de tipo mundial, ya que siempre ha estado presente y se encuentra en todas las sociedades sin importar el grado de desarrollo que tenga cada una de ellas. (Organización de las Naciones Unidas, 2014)

En Latinoamérica, la Organización Mundial de la Salud (OMS), la Comisión Económica para América Latina y el Caribe (CEPAL) o la Organización de Naciones Unidas (ONU) han investigado la incidencia de este fenómeno en diferentes países. Entre las principales conclusiones obtenidas en un diagnóstico realizado por la Organización Panamericana de la Salud en 10 países latinoamericanos se destaca que la violencia constituye 
un grave problema social y de salud pública que incide radicalmente en la vida cotidiana de las mujeres. Dicha violencia se expresa y se agrava a través del desarrollo de relaciones abusivas de poder, del control de las relaciones de la víctima y de la impunidad de los agresores. (Boira et. al., 2016)

De tal forma la violencia no toma en cuenta diferencias de etnia, religión, edad o género, ni tampoco nivel socioeconómico. Se puede experimentar o encontrarla en el trabajo, en la calle, en la comunidad y hasta en hogar. (Cabrejas , 2011)

La Organización Internacional del Trabajo (OIT) reconoce a los problemas de maltrato y de violencia en el trabajo desde hace ya varios años, siendo el sector de salud uno de los más afectados. Esto es debido a que las profesiones con trato interpersonal son los que tienen más alto riesgo laboral para agresiones y violencia de todo tipo. (Mejia et. al., 2016)

La violencia contra la mujer constituye uno de los problemas sanitarios de mayor relevancia en los últimos tiempos; de acuerdo con la Organización de las Naciones Unidas, cada 18 segundos una mujer es maltratada en el mundo. (Cervantes-Sánchez et. al., 2016)

En muchas ciudades de México se ha destacado el auge de la industria maquiladora, constituyendo este sector un centro laboral importante para la mujer. Sin embargo se enfrenta a la problemática social, que trasciende el nivel organizacional, siendo este el de las mujeres que son violentadas familiarmente, repercutiendo este hecho en la productividad de la empresa maquiladora.

Lo anterior apoyado en el hecho de que en el estado de Chihuahua en el año del 2011, el 47\% de las mujeres mayores de 15 años y más han tenido al menos un incidente de violencia hacia ellas a lo largo de la relación con su última pareja. (Instituto Nacional de las Mujeres, 2011)

Es por ello que el objetivo principal del presente, es con la finalidad de determinar la viabilidad de implementar un programa de apoyo interno, dirigido a las mujeres que son víctimas de violencia familiar en una empresa del sector industrial de la $\mathrm{Cd}$. de Chihuahua, pues al estar en capacidad de implementar programas de ayuda preventiva y en su caso correctiva dirigidos a mujeres que se encuentren vulnerables por el hecho de ser violentadas, impacta no solamente en el autoestima de la mujer trabajadora, sino también en el buen desempeño laboral de la misma, viéndose afectada positivamente la productividad organizacional.

Si bien es cierto existen como se mencionó anteriormente un sin número de investigaciones y estudios acerca del tema de la mujer víctima de violencia familiar, que abordan las consecuencias que sufren las víctimas violentadas a nivel físico y psicológico, lo que ha llevado tanto a instituciones gubernamentales y no gubernamentales a la creación de 
instituciones dedicadas exclusivamente a la ayuda en todos los aspectos. (Secretaría de Gobernarción, 2012)

Por lo que se debe de poner especial atención en las mujeres que se dedican al trabajo fuera de casa, ya que cada vez son más las mujeres que laboran al servicio de un empleador, lo que indica sin duda alguna que día a día aumenta el riesgo de tener dentro de la organización mujeres víctimas de violencia, ya que a pesar de la existencia de instituciones dedicadas a la atención de este problema social, parece ser que no es suficiente, pues no se ha logrado erradicar tal conducta, por lo que si dichas instituciones se vieran apoyadas con el sector privado laboralmente hablando, se pudiera llegar a concientizar y empoderar a las mujeres para que eviten el ser víctimas de violencia y lograr así el equilibrio social y laboral, impactando con esto en los índices de productividad de las organizaciones.

Por lo que genera una gran oportunidad de beneficiar con los resultados de la presente investigación a las organizaciones que estén interesadas en implementar nuevos programas de ayuda preventiva $y$ correctiva a las mujeres violentadas.

\section{Métodos, técnicas y procedimientos lógicos del pensamiento científico}

La investigación se llevó a a cabo de abril del 2014 a noviembre del 2015, en una empresa del sector maquilador de la Cd. de Chihuahua, con la ayuda del personal del departamento de recursos humanos.

La naturaleza de la Investigación fue mixta inductivo pues comprende por un lado la naturaleza cuantitativa pues se ahondó en el problema específico y que pudo explicar el fenómeno social de la violencia familiar, repercutiendo la misma en el contexto laboral. Por lado el aspecto cuantitativo con el fin de conocer los datos estadísticos a nivel local, nacional o internacional existentes referentes a la problemática.

Se trata de una investigación de forma explicativa que versa sobre la identificación de las causas de la baja productividad en las mujeres víctimas de violencia familiar, los efectos físicos, emocionales o psicológicos que se producen en ellas a tal grado que se puede ver afectada su estabilidad laboral.

Es tipo de investigación aplicada toda vez que se determinó la factibilidad de implementar programas de apoyo dentro de la organización a partir de los resultados que se orienten a la implementación de acciones de información, educación y comunicación para la prevención y erradicación de violencia familiar contra la mujer.

El diseño utilizado fue no experimental toda vez que únicamente se conocieron los hechos tal y como acontecieron, sin posibilidad de manipular variables. 
Es transeccional en virtud de que el tiempo de realización se pudo flexibilizar, sin embargo se realizó en un solo momento.

Para facilitar la realización de las entrevistas, se contactó al encargado del departamento de recursos humanos de la empresa analizada, asi mismo se aplicó el instrumento de medición al grupo focal (mujeres empleadas de dicha organización).

El procedimiento utilizado es el caso de estudio de la empresa seleccionada, toda vez que el cuestionario se aplicó única y exclusivamente a mujeres trabajadoras de dicho centro, que laboran en el segundo turno, constituyendo las mismas el grupo focal de la investigación, con lo que se pudo obtener datos estadísticos referentes a las mujeres víctimas de violencia familiar dentro de la organización referida.

La investigación es hermenéutica pues enfatiza la comprensión e interpretación de las variables independientes manejadas en el maltrato familiar y la variable dependiente que afecta la productividad laboral de las mujeres víctimas de violencia.

Las variables del caso de estudio que se evaluaron fueron:

1.- Los generales de la empledas como: la edad, estado civil, puesto que ocupa.

2.- La violencia familiar que se pude presentar de diferentes formas ya sea: sexual, física, psicologica o financiera.

3.- La solicitud de ayuda a organimos públicos para solicitar diferentes tipos de apoyo, pero sin llegar necesariamente a la denuncia, como lo son la ayuda de tipo: legal, médica y psicológica.

4. La denuncia de la violencia familiar.

5.- La afectación al trabajo que se puede ver reflejado en retardos, faltas injustificadas, permisos, recision de contrato, cambio de tuno, accidentes laborales, renuncias son consecuencia de la violencia familiar.

6.- Los costos por ausentismos

La población es de 1630 mujeres.. Con una muestra de 65 mujeres encuestadas, $\mathrm{y}$ donde $\mathrm{K}=1.65$ con un nivel de confinanza del $90 \%$, error muestral del 10\%, la proporción de mujeres s que poseen en la población la característica de estudio que se estimo que son violentadas.

La selección de la muestra se realizó de manera aleatoria. El instrumento de medición para recolectar la información de campo fue el cuestionario, de preguntas cerradas con varias opciones de respuesta. Es un instrumento de medición estandarizado toda vez que se midieron las consecuencias laborales de ser violentada familiarmente la mujer.

\section{Resultados}

Se llegaron a los resultados de dos formas, una através del instrumento y la otra a travéz de las investigación directa en instancias 
públicas para la elaboración de una propuesta de prevención de este hecho estudiado.

A continuación se detallan los resultados encontrados por constructo a través de la aplicación del instrumento previamente evaluado tanto interno como externamente:

El 50\% de las empledas encuestadas oscilan entre los 30 y 40 años de edad, siendo este el promedio de edad de las empleadas de la empresa analizada y por lo tanto el promedio de edad de la mujer que vive con violencia.

Así mismo el 51\% de las empleadas son solteras, considerando en este porcentaje tanto a las empleadas que están solteras como a las divorcidas. Contra un $49 \%$ de empledas que sé encuentran casadas o en unión libre.

Cabe mencionar que un $87 \%$ de las empleadas son de puestos de nivel operativo y un $13 \%$ de puestos administrativo.

De las 65 mujeres trabajadoras, 48 de ellas sufren violencia familiar, que corresponde a un 73\% que son la gran mayoría , contra 17 empleadas que equivale a un $26 \%$ que manifiestan no haber vivido esta problemática social.

De las mujeres que manifestaron vivir con violencia 21 mujeres dijeron vivir la violencia física, 35 mujeres la violencia psicológica, 3 violencia sexual y 10 mujeres violencia económica. Realmente el 34\% de las empleadas viven con violencia física pues la violencia sexual, puede ser considerada como tal.

Sólo el 21\% de las empledas encuestadas ha recibido ayuda del algún organismo público como el Instituto Chihuahuense de la Mujer o de la Fiscalía Especializada en delitos contra la Mujer. Realmente es muy bajo el procentaje de la mujer que solicita ayuda o apoyo. Del 21\% de mujeres que han recibido ayuda, el 51\% ha recibido ayuda psicológica, $18 \%$ ha sido ayuda médica, y 31\% ayuda de tipo legal.

De las empleadas que viven con violencia física, el $76 \%$ manifiesta haber recibido lesiones, este porcentaje es muy alto, pues a pesar de las campañas de género y de no violencia a la mujer por parte de organizaciones públicas, aún no se ve impactado de forma sobresaliente. Aunado a lo anterior que sólo el 29\% de las empleadas denuncian el maltrato.

El 79\% de las mujeres acuden a laborar después de haber sido violentadas. De lo que se puede deducir que el grado de responsabilidad hacia el trabajo de la mujer es alto.

El 89\% de las mujeres consideran que la violencia familia afecta su trabajo. Ese porcentaje se se ve reflejado en un $18 \%$ en incapacidades, un $25 \%$ en solicitud de permisos, un $26 \%$ en faltas injustificadas, un $9 \%$ en 
accidentes de trabajo, un 9\% en recisión de contratos y un 13\% en cambio de turno.

El 81\% de las empleadas encuestadas consideran que disminuye su producción después de ser violentadas.

Sólo el 25\% de las empleadas que vivien violencia familiar mencionan haber recibido ayuda o algún tipo de apoyo de la empresa a la cual laboran y que se analiza en el presente estudio. La totalidad de ellas consideran que les gustaría que la empresa contará con algún tipo de programa de apoyo contra la violencia familiar.

Sé calculó el análisis de confiabilidad para el instrumento por constructo, a través del Alfa de Cronbach con un 0.914, dando como resultados un Alfa de Cronbach basado en los items tipificados de 0.918, valor que puede ser aceptado para los 18 items analizados.

\section{Análisis de correlación para el instrumento por constructos}

En el análisis correlacional se encontraron relaciones entre las variables medidas por el instrumento, la relación entre ellas se describe a continuación:

La edad y estado civil se relacionan con la violencia fisica y sexual, ya que se determinó en una relación directa del 80.5\%. Como así sucede los casos de violencia familiar en la mujer, se observó en casos en que la mujer se encuentra casada o vive en unión libre, de cualquiera de las dos formas, es en el caso que la mujer tiene una pareja. Prefetentemente entre los 30 y 40 años de edad.

Asi mismo el estado civil se relaciona con la violencia psicologica, pues se encontró una relación de 85.6\%. Que como en el punto anterior la violencia psiologica se da en los casos en que la mujer tiene una pareja sentimental.

Existe una relación de $86.8 \%$ de correlación entre el puesto y al variable de la violencia familiar, lo que que indica que cuando las mujer ocupa un puesto operativo es más vulnerable a sufrir cualquier tipo de violencia familiar.

Se puede decir que la violencia física y la violencia sexual se encuentran relacionadas con la variable de solicitud de ayuda, pues existe una relacion de $91.7 \%$. Indiscutiblemente la solicitud de ayuda y/o apoyo a organismos públicos institucionales se da cuando la mujer es violentada fisica y sexualmente, lamentablemente sólo en esos casos, quedando sin considerar los diferentes tipos de violencia familiar que existen.

La violencia física y la violencia sexual se relacionan con la denuncia, pues se encontró una relación del 89.05\%, pués al igual que el párrafo anterior, la mujer violentada sólo cuando es agredida en su persona se acerca a la autoridad correspondiente para denunciar este hecho. 
La violencia física y la violencia sexual se relacionan con con las variables de permisos, faltas injustificadas, cambio de turno, recisión de contrato, ya que se encontró una relación del $87.2 \%$. Es inevitable que la mujer al ser violentada en su persona deje de ser productiva, reflejandose esto principalmente en el ausentismo al trabajo de alguna u otra manera, perjudicando a la empresa a la cual labora en sus índices de productividad.

Como se describe en el cuandro No. 1 donde se muestra un comparativo de mujeres trabajadoras con y sin violencia, que resulta ser información comprobatoria, en donde el departamento de recursos humanos de la organización estudiada, proporcionó información relevante de los casos previamente identificados de mujeres víctimas de violencia familiar. $\mathrm{Al}$ realizar una revisión del expediente personal de las empleadas, se reflejó que la totalidad incide principalmente en faltas injustificadas y permisos, tal como se muestra en dicho cuadro, en donde se puede observar y comprobar plenamente las correlaciones antes explicadas, principalmente la de violencia con faltas injustificadas.

\section{Conclusion}

Los resultados arrojados por el cuestionario aplicado a la muestra femenina en la empresa analizada, evidenció que existen más casos de los que se conocían de violencia familiar en las empeladas de dicha empresa, y si bien es cierto que el departamento de recursos humanos tiene identificados plenamente algunos casos, se ignoraba que la mayoría de su población femenina sufriera de violencia familiar y que considerara la implementación de algún programa de apoyo tendiente a contrarrestar la problemática social dentro de la organización.

Así mismo con la aplicación del instrumento de medición, se concluye que las consecuencias laborales de las empleadas que viven violencia familiar resultaron ser: bajo desempeño en las actividades laborales, accidentes de trabajo derivados del estrés o distracciones por estado anímico de las empleadas, así como el ausentismo en el cual se encuentran inmersos tanto las faltas injustificadas, los permisos y las incapacidades; por otro lado cambios de turno y por último las rescisiones o renuncias que han experimentado en empleos anteriores a causa de la violencia familiar en que viven.

Ahora bien, como se puede apreciar en la información contenida en el cuadro No. 1, donde se puede calcular los costos que impactan a la organización de las mujeres que son víctimas de violencia familiar, para esto sólo se tomará en cuenta las faltas injustificadas y los permisos, ya que las incapacidades no son una fuente objetiva, pues se desconoce si realmente fue causada por violencia familiar o por alguna enfermedad general, pues el departamento de enfermería se limita a clasificar las incapacidades bajo dos 
rubros: por riesgos de trabajo o enfermedad general, incluyéndose en esta última cualquier evento distinto a riesgos de trabajo.

Cuadro 1. Comparativo de mujeres trabajadoras con y sin violencia familiar

\begin{tabular}{|c|c|c|c|c|c|}
\hline \multicolumn{2}{|c|}{ Empleadas que viven violencia familiar } & \multicolumn{3}{c|}{ Empleadas sin violencia familiar } \\
\hline No. de Empleada & Faltas & Permisos & No. de Empleada & Faltas & Permisos \\
\hline 15454 & 2 & 2 & 10786 & 0 & 0 \\
\hline 12752 & 5 & 0 & 10990 & 0 & 1 \\
\hline 14244 & 6 & 0 & 11033 & 0 & 0 \\
\hline 11321 & 1 & 8 & 09970 & 0 & 0 \\
\hline 16696 & 3 & 0 & 10126 & 1 & 0 \\
\hline 9578 & 3 & 4 & 16509 & 0 & 0 \\
\hline 13421 & 5 & 1 & 13584 & 0 & 2 \\
\hline 12539 & 3 & 2 & 11214 & 0 & 0 \\
\hline 12899 & 2 & 5 & 15987 & 1 & 0 \\
\hline 5673 & 1 & 2 & 11690 & 0 & 1 \\
\hline 13222 & 4 & 4 & 10250 & 2 & 0 \\
\hline 10665 & 3 & 1 & 16777 & 1 & 0 \\
\hline 15852 & 2 & 6 & 8236 & 0 & 1 \\
\hline 13212 & 6 & 0 & 11001 & 0 & 0 \\
\hline 7715 & 4 & 3 & 14339 & 0 & 1 \\
\hline
\end{tabular}

Fuente: (Elaboración propia, 2015)

Para el cálculo de la pérdida económica estimada en la organización que es generada por las empleadas víctimas de violencia familiar se toma en cuenta los siguientes factores:

Producción: 60 lentes por hora

Costo por lente: 8 dólares

Horas diarias productivas: 8 horas

Faltas injustificadas de mujeres victimas de violencia : 48 días $=384$ horas hombre.

Permisos de mujeres victimas de violencia: 39 (19.5 días) = 156 horas.

Total de horas perdidas en un período de 6 meses por faltas y permisos: 536 horas.

De lo anterior en un período de seis meses se dejaron de producir 32,160 lentes y partiendo de la base que el costo por lente es de $\$ 8$ dólares, esto arroja un total de $\$ 257,280$ dólares perdidos por falta de producción de 15 mujeres víctimas de violencia que incurrieron en faltas y permisos en dicho período.

Continuando en este orden de ideas, es menester señalar que los programas de apoyo que ofrecen tanto el Instituto Chihuahuense de la Mujer como la Fiscalía Especializada en atención a mujeres víctimas del delito por razón de género consisten en apoyo médico, psicológico, jurídico o legal, terapéutico, educativo, social y comunitario; y si bien es cierto se cuenta con que las instituciones gubernamentales como el Instituto Chihuahuense de la 
Mujer y la Fiscalía Especializada en atención a mujeres víctimas del delito por razón de género, según la información proporcionada por las propias instituciones atienden en forma gratuita a las mujeres víctimas de violencia familiar, brindándoles el apoyo antes citado.

También de acuerdo a los resultados arrojados, más de la mitad de las mujeres encuestadas y que sufren violencia no acuden a denunciar los hechos, por lo que en consecuencia es necesario concientizar y empoderar a la mujer para que acuda a las instituciones a denunciar la violencia y recibir el tipo de ayuda que requiera cada caso en especial.

Es importante mencionar que muchas de las mujeres violentadas no realizan las acciones necesarias para evitar seguir siendo víctima, debido al hecho de que ello representa tener que ausentarse de sus labores por algunos períodos de tiempo, lo que afecta directamente la economía familiar, por lo que para para ellas es una prioridad presentarse a sus labores en lugar de acudir a denunciar un hecho violento, inclusive lo pueden llegar a ver como parte de su vida diaria, lo cual repercute en los costos de la organización, ya que al acudir a laborar después de un episodio violento, la mujer trabajadora y como ella misma lo confirma con el instrumento de medición al que respondió en la mayoría de los casos que si afecta o se ve disminuida su productividad, llegado inclusive al extremos de tener que renunciar a sus empleos o bien en el peor de los casos las organización le rescinde su contrato de trabajo, por lo general por haber acumulado más de tres faltas en un período de treinta días.

En relación a todas las conclusiones ya mencionadas y al alto índice encontrado en un $73 \%$ del personal femenino principalmente de nivel operativo sufre de violencia familiar; y en la demanda que manifiestan las mujeres de la gran necesidad que existe de implementar programas de apoyo a la variable analizada, es por ello que en el siguiente apartado de recomendaciones se presenta un programa que de implementarse le resultaría a la mujer violentada de gran beneficio tanto en su vida personal como laboral

\section{Recomendaciones}

En base a los resultados que se obtuvieron del instrumento de medición, en el último item la totalidad de las mujeres encuestadas comentaron que SI les gustaría que la organización estudiada, contara con un programa de ayuda para contrarrestar la violencia familiar.

Por lo anterior se presenta la siguiente propuesta, basada principalmente en la demanda anterior y en los apoyos que otorgan las asociaciones gubernamentales y civiles ya existentes.

A continuación se describen programas de apoyo que ofrecen las instituciones no gubernamentales de la $\mathrm{Cd}$. de Chihuahua, dirigidos a las 
mujeres que viven la violencia familiar, los cuales deben de ser difundidos principalmente por las oragnizaciones a sus empleadas.

El Instituto Chihuahuense de la Mujer: Ofrece programas de atención psicológica, asesoría legal, canalización para la atención médica, asesoría en procesos productivos, cursos, talleres y seminarios de prevención.

El Consejo Ciudadano: Ofrece programas con talleres de prevención de violencia familiar, canalización, acompañamiento y seguimiento a casos de violencia familiar.

La Casa Comunitaria Juana de Abaje: Ofrece programas con talleres titulado "Compartiendo realidades", cine club, ejercicios de relajación (anti gimnasia) y talleres de fotografía.

A continuación se describe una propuesta de prevención de violencia social para implementarse dentro de la empresa analizada:

\section{Capacitación}

Objetivo: Otorgar pláticas semestralmente sobre las consecuencias que se viven cuando la familia este inmersa en la problemática social de la Violencia familiar.

Temas: ¿Qué es la violencia familiar? , tipos de violencia, impacto de la violencia familiar: Daño físico, psicológico, etc..., ¿Cómo y quién genera la violencia familiar en el hogar?, factores externos que influyen en la violencia familiar, la importancia de la denuncia por tratarse de un delito, ¿Cómo acudir a interponer una denuncia?

Instituto que ofrece el curso: Instituto Chihuahuense de la Mujer

Inversión: Las instituciones antes descritas otorgan apoyos totalmente gratuitos. La empresa pudiera ofrecer estas capacitaciones, ya sea dentro del horario de trabajo o fuera de el.

\section{Cine club}

Objetivo: Otorgar a los empleados un espacio de esparcimiento por medio de películas con temas en valores familiares, para generar la reflexión no sólo de los empleados también puede cualquier miembro de la familia de los trabajadores.

Instituto que lo ofrece: Casa Comunitaria Juana de Asbaje

Inversión: Espacio tal como una sala de capacitación que cuente con audiovisual (bocinas y proyector). Se sugiere que para esta actividad la empresa facilite una hora productiva y la otra hora la ponga el trabajador fuera de su horario laboral. O bien que la empresa proponga los horarios más adecuados si decide la participación de la familia del empleado. 
Propuestas correctivas: Basándose en los resultados en que el tipo de violencia más común que vive la mujer trabajadora, es la de tipo psicológico, por tal razón la propuesta irá enfocada en este rubro.

\section{Módulo de atención}

Objetivo: Otorgar asesoría y orientación a la empleada que viva en una problemática social, tal como la violencia familiar, a través de un psicólogo, de un abogado y/o de un especialista en el tema.

Mecánica: Publicar o difundir la instalación de un módulo de atención para que los empleados puedan ser asesorados de cualquier problemática social (incluyendo violencia familiar) que esté viviendo, con el fin de ser atendidos por personal especializado y que se tenga un registro de los casos, así como el seguimiento de cada problema social que manifiesta él o la empleada de la empresa.

En esta propuesta es recomendable que se atienda cualquier problemática social que afecta a las empleadas, ya que si se puede limitar solamente a la atención para la violencia familiar, puede haber el riesgo que el personal no se acerque por temor o vergüenza a ser identificado como víctima de la violencia.

Inversión: Espacio para instalar el módulo de atención, de preferencia un espacio donde haya privacidad.

Institución que lo ofrece: La casa comunitaria quien es la que facilita al gremio de la industria maquiladora una psicóloga que pueda acudir un día a la semana a la empresa que lo solicite. La psicóloga durante su estancia en la organización, analizará los casos de las personas que le solicitan ayuda, y realizará las gestiones necesarias a efecto de canalizar a la víctima a la institución más adecuada de conformidad con la gravedad de la problemática que presenta la empleada. Este recurso (la psicóloga en la organización) no genera ningún costo para la empresa, ya que es parte de los servicios que ofrece la Casa Comunitaria.

\section{Atención en línea}

Objetivo: Establecer un enlace entre los empleados de la empresa y autoridades de manera sencilla para fomentar la denuncia y agilizar los trámites relacionados a ella. Los empleados podrán hablar directamente al *2232 y recibir asesoría jurídica y psicológica en caso de ser víctimas de violencia.

Mecánica: Las empleadas víctimas de violencia deberán llamar telefónicamente al *2232, posteriormente será entendida por un especialista (psicólogo o abogado) vía telefónica, el cual atenderá el caso, asimismo dará apoyo de acompañamiento en caso de requerirlo la empleada y otorgar el reporte sobre el estatus del caso a la gerencia de recursos humanos, con el 
propósito concienticen sobre la problemática de la víctima a fin de contar con la flexibilidad de la empresa en cuanto a retardos o faltas de la empleada, cuando la misma tuviera alguna cita en horario de labores, previa comprobación de que efectivamente se encontraba en algún trámite relacionado con la problemática de la violencia.

Inversión: Para efectos del presente apoyo se requiere que la organización cuente en las instalaciones de la misma con teléfonos públicos, lo que se validó que en la empresa estudiada se cuenta con dicha infraestructura. Las horas hombre generadas por ausencias de la empleada al acudir a sus citas, cuando se realicen en horario de trabajo.

Propuesta de Difusión: Se sugiere que la organización se comprometa a la difusión y publicación masiva, en lugares visibles de los programas que desee implementar, a fin de que la empleada pueda acercarse a los programas previamente elegidos con la confianza de que obtendrá la ayuda requerida en forma confidencial y de esta forma se logre disminuir el impacto en la productividad laboral en las mujeres que son víctimas de violencia.

\section{References:}

Boira, S., Carbajosa, P. y Méndez, R. (2016). Miedo, conformidad y silencio. La violencia en las relaciones de pareja en áreas rurales de Ecuador. Psychosocial Intervention 25(2016) 9-17. http://web.a.ebscohost.com/ehost/pdfviewer/pdfviewer?sid=c437c839-95034f5b-ac5a-921c236fd060\%40sessionmgr4010\&vid=41\&hid=4206

Cabrejas, M. (Julio, 2011). Violencia Contra las Mujeres. Noviembre 17, 2015, de Asamblea General de Naciones Unidas Sitio web: www.riicotec.org/.../groups/.../col2011_violencia contra las mujeres repercusiones sociales y sanitarias

Cervantes-Sánchez, P., Delgado-Quiñones, E., Nuño-Donlucas, M., Sahagún-Cuevas, M., Hernández-Calderón, J. y Ramírez-Ramos, J. (2016). Prevalencia de violencia intrafamiliar en embarazadas de 20 a 35 años de una de unidad medicina familiar. http://web.a.ebscohost.com/ehost/pdfviewer/pdfviewer?sid=c437c839-95034f5b-ac5a-921c236fd060\%40sessionmgr4010\&vid=82\&hid=4206 Instituto Nacional de las Mujeres. (Enero 16, 2014). Estadística de la Violencia contra las Mujeres en México. Noviembre 19, 2015, de Inmujeres. gob.mx Sitio web: http://estadistica.inmujeres.gob.mx/formas/convenciones/Nota.pdf Mejia, R., Allpas H., Caceres O. y Peru, R. (2016). iMedPub Journals. Archivos de medicina. ISSN 1698-9465. Vol. 12 No. 1: 4. Doi: 10.3823/1280. http://web.a.ebscohost.com/ehost/pdfviewer/pdfviewer?sid=c437c839-95034f5b-ac5a-921c236fd060\%40sessionmgr4010\&vid=56\&hid=4206 
Organización de las Naciones Unidas. (2014)._Informe de la Conferencia Mundial para el examen y la evaluación de los logros del decenio de las naciones unidas para la mujer: Igualdad, desarrollo y paz, Publicación de las Naciones Unidas. Capit. IV, Secc. C, párrafo 229.

Secretaría de Educación Pública. (2000). Violencia en la Familia. México. D.F.: Talleres Gráficos. “p”. 4

Secretaria de Gobernación. (2012). Violencia Emocional, Fisca, Sexual y Económica. Noviembre 17, 2015, de CANAPO Sitio web: http://www.violenciaenlafamilia.conapo.gob.mx/en/Violencia_Familiar/Viol encia_emocional_fsica_sexual_y_economica 